\title{
Localization Applications of 3D-GIS Artificial Weather Modification Operational Command System in Fuxin, China
}

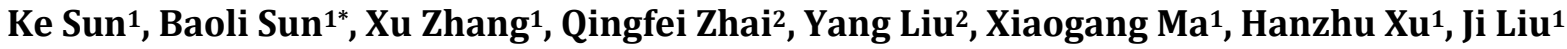 \\ ${ }^{1}$ Fuxin City Meteorological Bureau, Fuxin, China \\ ${ }^{2}$ Liaoning Province Meteorological Bureau, Shenyang, China \\ Email: *sunkel2020@163.com
}

How to cite this paper: Sun, K., Sun, B. L., Zhang, X., Zhai, Q. F., Liu, Y., Ma, X. G., Xu, H. Z., \& Liu, J. (2019). Localization Applications of 3D-GIS Artificial Weather Modification Operational Command System in Fuxin, China. Journal of Geoscience and Environment Protection, 7, 125-134. https://doi.org/10.4236/gep.2019.76011

Received: November 26, 2018

Accepted: June 24, 2019

Published: June 27, 2019

Copyright $\odot 2019$ by author(s) and Scientific Research Publishing Inc. This work is licensed under the Creative Commons Attribution International License (CC BY 4.0).

http://creativecommons.org/licenses/by/4.0/ (C) (i) Open Access

\begin{abstract}
Based on the command and management requirements of weather modification operations in Fuxin City, China, a new generation of three-dimensional operation command system platform for new artificial weather modification was developed and developed using modern communication network technology. The system uses integrated three-dimensional geographic information system (3D-GIS), global positioning system (GPS) positioning and virtual reality technology to calculate, analyze and process the new basic geographic information and weather modification information vector data, generate real-time weather modification operations and guide products in Fuxin area, and realize the impact on labor. The three-dimensional, comprehensive and effective management of basic weather information, early warning and forecast information, disaster prevention and mitigation information and other decision support information provides technical support for scientifically and effectively managing and directing weather modification operations.
\end{abstract}

\section{Keywords}

3D-GIS, Artificial Weather Modification, Disaster Prevention and Mitigation

\section{Introduction}

Artificial weather modification, hereinafter referred to as "weather modification", refers to the activities that humans use modern science and technology to exert influence on the local atmosphere and achieve the purpose of increasing rain, preventing flooding, eliminating rain, and eliminating fog (Xu \& Yin, 2017; Sin'kevich et al., 2018). Its purpose is to use scientific methods and techniques to 
take advantage of favorable natural climate and weather conditions to influence different objects to increase or decrease precipitation, suppress hail, eliminate fog, and prevent frost (Xiao, 2007).

In recent years, the emphasis on and research on weather disasters, and the gradual maturity of theory and technology, have greatly improved people's ability to observe, understand and predict the weather (Zeng, 2006), providing the necessary theoretical and technical basis for weather modification work. The information development and artificial decision-making of artificial precipitation enhancement and prevention, as well as flood control has made the weather modification as an important part of disaster prevention and mitigation, and has been paid more and more attention by governments and people at all levels (Huang, 2004). Weather modification operations involve a variety of information such as meteorology, satellite remote sensing, geographic information, and social economy. These types of information are complex, and are mostly related to geospatial information. In order to accurately locate and display these data, comprehensive analysis and processing are carried out. Provide reference to decision makers, especially the support of GIS (Huang et al., 2007). Therefore, the application of GIS (Geographic Information System) to weather modification plays an important role in guiding weather modification operations ( $\mathrm{Li}, 2005)$.

With the rapid development of computer technology and data acquisition technology, the development of three-dimensional visual virtual simulation geographic information system, namely the development of three-dimensional geographic information system (3D-GIS), integrated with remote sensing (RS), geographic information system (GIS) and virtual reality (VR) has attracted much attention (Elsahabi \& Negm, 2017; Ujang \& Rahman, 2013). 3D-GIS uses three-dimensional data to describe geographic objects; that is, all objects are represented by three-dimensional coordinates $(\mathrm{X}, \mathrm{Y}, \mathrm{Z}$ or latitude and longitude and altitude), such as resources, environment, meteorology, city, ocean, military and other major issues. The social application service for solving and spatial information urgently needs the support of 3D-GIS.

It is reported in the literature (Strelau et al., 2013; Li et al., 2017; Wang et al., 2017) that there are many applications of the city-level weather modification operation command system based on traditional GIS; however, the application of 3D-GIS is rare. Based on 3D-GIS, GPS and virtual reality technology, this paper has developed a 3D weather modification command system, which is applied in the weather modification operation to provide technical support for the scientific command of the shadow operation, which makes the figure operation more reasonable and effective.

\section{Methods}

The 3D weather modification operation command system integrates 3D geographic information, GPS positioning and virtual reality technology. It uses different scale digital elevation model (DEM) terrain data and remote sensing im- 
ages with different resolutions to establish a $3 \mathrm{D}$ geographic information platform with different scales, landforms and ground truth. On the basis of the three-dimensional geographic information system, the basic geographic information and the avatar information vector data are added to visually display the distribution of the operation point, the distribution of the river, the distribution of the key rainfall-increasing areas, and the meteorological observations. Through the development and integration of systems based on massive data, the integrated management of basic business information, early warning and forecast information, job command information, job analysis and monitoring information is realized. Finally, this system will be developed into a 3D weather modification command system platform integrating data and products display, early warning, live monitoring, aircraft tracking and other management functions. It will provide more intuitive and accurate information services for weather modification management in Fuxin City, China.

\subsection{System Structure}

The main functions of the system include three-dimensional display analysis of radar and cloud image, display and analysis of various data such as numerical products, sounding, ground truth, rain and flood control operations, mobile rocket vehicle monitoring, and aircraft operation tracking (see Figure 1).

Based on the $90 \mathrm{~m}$ geographic information elevation image data and the $14 \mathrm{~m}$ precision satellite image data, the system completes the realistic display of $3 \mathrm{D}$ geographic information. After the superposition and display of the two-dimensional geographic information data, the various geographic information layers that the business needs to display can be superimposed and displayed, and the close connection between the three-dimensional imaging technology and the meteorological industry is realized.

The system directly calls the Doppler radar echo and the digital radar echo to complete the three-dimensional display of the radar echo product. At the same time, horizontal and vertical section display at any angle can be realized for 3D images. In addition to a variety of satellite cloud image display, the system can

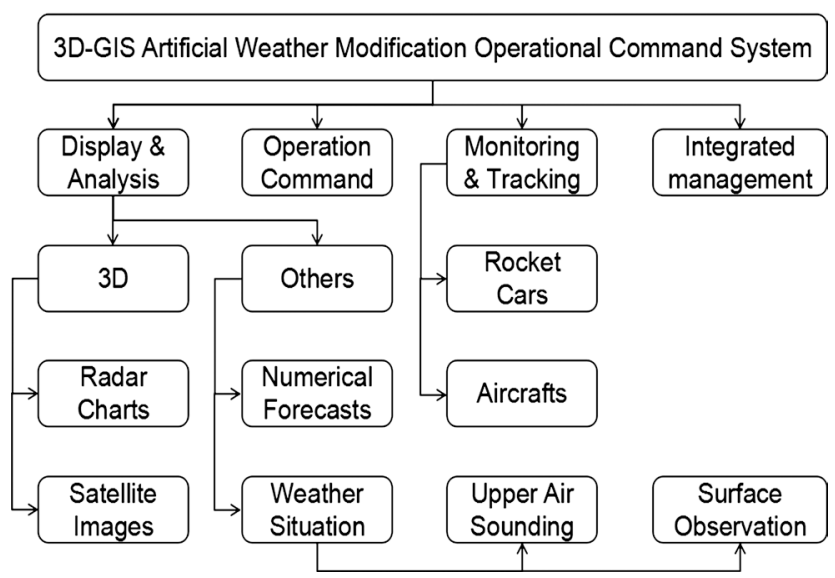

Figure 1. Structure diagram for the $3 \mathrm{D}$ weather modification command system. 
also support the processing of three-dimensional display of satellite imagery. The system can be connected to the automatic station database, which can display the position and live data of the automatic station in real time. At the same time, it can provide different levels of color warning for the automatic station rainfall, and support the three-dimensional color patch display of MICAPS sounding observation data and grid data. Such as high temperature field, humidity field, convection field and other data display.

For the display of meteorological data products, the system not only developed the display functions of meteorological data such as radar, cloud map, lightning, automatic station, rainfall, ground high-altitude, etc., but also developed a variety of phantom product query display, taking into account various meteorological data. The three-dimensional crossover effect enables the commander to fully grasp the overall situation and provide comprehensive capabilities for data analysis. Through comprehensive analysis of various related materials, we strive to achieve reasonable catalysis, joint operations, overall arrangement, and scientific figures.

\subsection{Radar Echo Analysis}

The radar echo analysis function interface includes 8 analysis products such as single-layer PPI display, multi-layer PPI display, vertical section, horizontal section, vertical liquid water content, echo top height, and echo combined reflectivity, and can also perform 3D radar. The echo product portfolio is analyzed for overlay. The 3D radar echo provides two layers of 3D image display of different intensities, which are called inner 3D echo and outer 3D echo image. The inner layer is a strong echo 3D image with an intensity of $35-65 \mathrm{dBz}$, and the outer layer $3 \mathrm{D}$ is a weak echo $3 \mathrm{D}$ display with an intensity of $5-45 \mathrm{dBz}$. The commander can choose to display a single strong echo or a single weak echo, or simultaneously display two intensity echoes for translucent overlay display.

The radar echo profile is divided into horizontal section and vertical section. The former is generated by interpolation of PPI linear data at different elevation angles in the same azimuth. The vertical section is the vertical profile of radar echo generated between any two points on the map. The realization of the horizontal section refers to selecting an angle around the center of the radar. When a certain angle is selected, the system performs horizontal section calculation and display between the radar center and the position of the user's mouse point.

The horizontal profile implementation algorithm is now described in detail. The horizontal profile is the intensity of the PPI at different elevation angles in the vertical direction. The value is taken as the measured point, and the values of other pixels in the horizontal section are obtained by the bilinear interpolation and the distance weighted average algorithm by the PPI intensity values of the adjacent two layers. Assuming $\mathrm{V}$ represents a different elevation angle, and point $P$ is not at any elevation angle, the value of point $P$ needs to be calculated by the measured values $P 1, P 2, P 3, P 4$ on the upper and lower elevation angles V1, V2. 
Then the formula for calculating the intensity of the $P$ point is:

$$
P=(P 1 * L 3+P 2 * L 4+P 3 * L 1+P 4 * L 2) /(L 1+L 2+L 3+L 4)
$$

When calculating the positions of $P, P 1, P 2, P 3$, and $P 4$, the curve of the curvature of the earth and the radar echo in the atmosphere must be taken into consideration, and the spatial position calculation is performed by using the radar equation, so as to ensure that the obtained profile data is objective and accurate. In addition, when processing the interpolation, if the values of the two adjacent elevation angles are NULL values, the value of the relevant direction needs to be canceled from the formula, and at least two values should be guaranteed to be non-Null values, and the formula can be calculated. If only one adjacent $P$ value is non-null, you can choose to calculate the other side as zero.

\subsection{Three-Dimensional Display of Artificial Rainfall Aircraft Routes}

The called aircraft route data configuration file is in text format (e.g. fjhx.txt), including information such as latitude and longitude, altitude, speed, heading, temperature and humidity. After the route data is read, the spatial linear data of the three-dimensional geographic information system is formed, and the $3 \mathrm{D}$ digital model of the Fuxin rain-increasing aircraft is created by using the $3 \mathrm{D}$ model tool, and converted into a three-dimensional geographic information system model format, which is completed in the three-dimensional geographic information. display. When the aircraft operation simulation is realized, the position and posture of the aircraft are continuously updated, and the three-dimensional live display of the working aircraft is realized. Through the operation monitoring system, the trajectory of the aircraft operation is obtained, and the real-time operation command is carried out, which provides the ground commander with the possibility of flight monitoring and command of the aircraft operation, and also improves the efficiency of the rain-increasing operation. Through the system call the module program code, the three-dimensional dynamic display of the aircraft air flight trajectory is realized. Part of the code is as follows:

BOOL Draw3DPlaneLine()

$\{$ POSITION pos = m_airLineList.GetHeadPosition();//Open route data link table

$$
\text { while (pos) }
$$

$\{\quad$ pGpsObj $=$ m_airLineList.GetNext(pos); //Obtain an object of an airplane

$$
\text { if (pGpsObj->MoveToNext()) //Drawing route }
$$

nodes

\{ pGpsObj->ShowSafeInfo(m_bISShowCircle); //Display safety distance circle

$$
\text { pGpsObj->ShowGpsInfo(m_bIsShowTip); //Information }
$$

bulletin board showing the aircraft

\} $\quad$ return TRUE;

\} 


\subsection{Operation Site's Warning}

When the radar echo reaches the early warning indicator, the system automatically calculates the working point that can be affected within 10 - 30 minutes. If the working point happens to be within the warning area, the working point will become flashing and the color of the working point text will change. It is green. Through the early warning management platform, the radar echo warning information is transmitted, including all the early warning operation points of rain enhancement and flood prevention. After the system automatically analyzes and judges, the location, working orientation, working height and working ammunition of each early warning operation point are automatically calculated. The warning job point becomes blinking and is implemented by the following code:

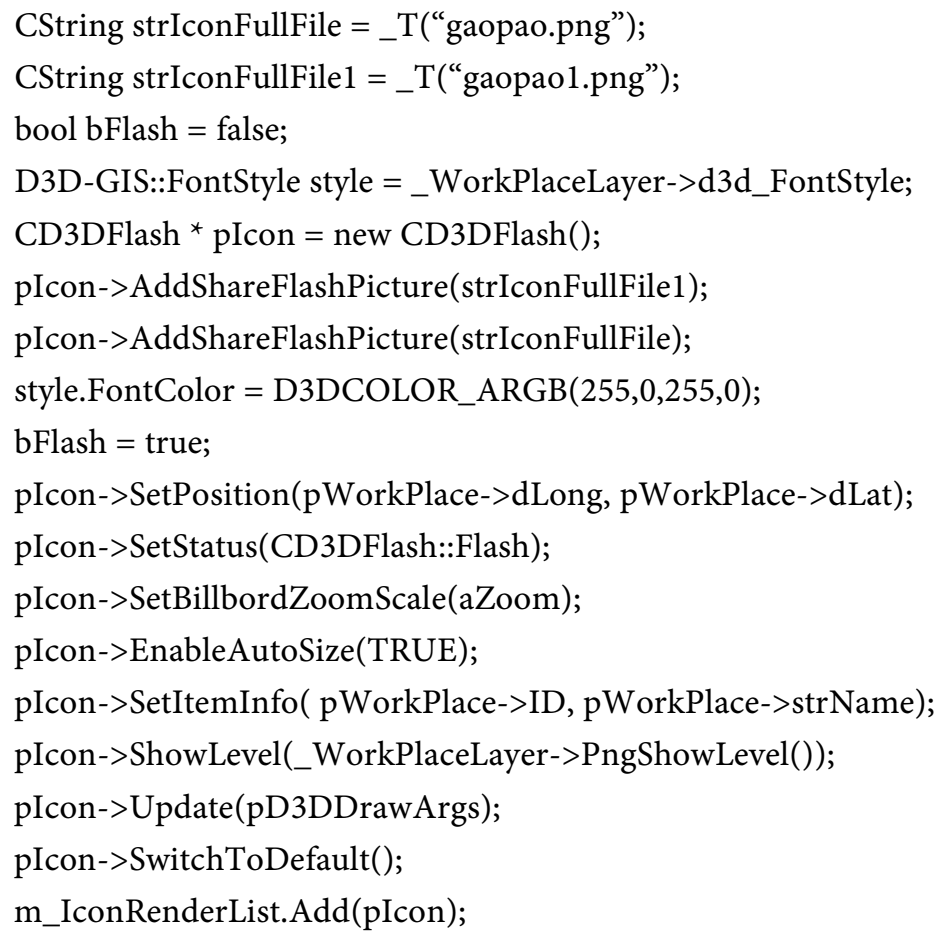

In addition, a new design of the job site warning text information that needs to be automatically converted to generate a Word document, including the warning release time, decision data time, weather situation map, job plan details, etc., is provided to the commander to initiate the operation warning. Finally, it is reserved for archiving information. The implementation of some of the code is as follows:

CString strTemplateFile;

strTemplate-

File.Format(_T(“\%s\Templatel\TestDot.dotx”),theApp.m_strCurPath);

m_pWord=new CWordOffice();

m_pWord->CreateDocuments();

m_pWord->CreateDocument(strTemplateFile);//Create a file from a template

m_pWord->ShowApp(); 


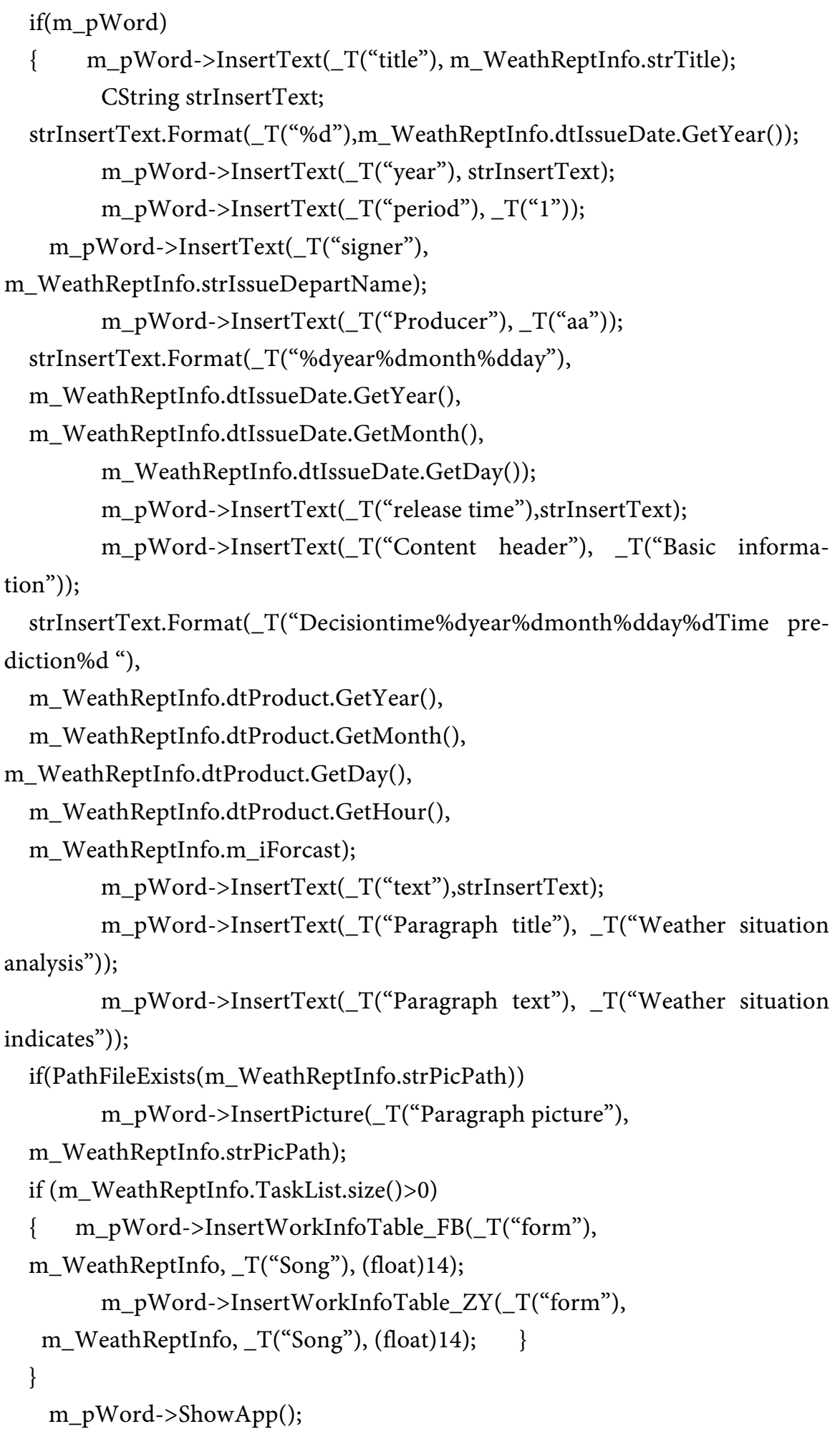

\subsection{Operation Management}

The figure command system realizes operator management, job site management, work equipment management, work ammunition management, and job live management through a database. 
Operator management includes commanders, operators, and managers. Under the site personnel menu, click on different categories to enter different management interfaces to add and modify personnel information. Job site management includes adding, deleting, and modifying site information, including site profiles, basic information, staffing, job status, multimedia information, and forbidden zone information. Work equipment management includes six categories of management of communication tools and work tools. Operational ammunition management includes functions such as ammunition type, inbound and outbound management, inventory inquiries, and ammunition statistics. The job live management includes functions such as job report, job information statistics, and job information query.

\section{Results and Discussion}

We now introduce an application example of artificial precipitation on June 7, 2014. At the same time, the field crops are entering the key growth period, and there is no effective precipitation for more than 20 consecutive days. The drought in most of the city's plots is very unfavorable for crop growth. On June 5, through the analysis of meteorological data, combined with the provincial and municipal weather forecasting consultations, the forecast results were obtained: affected by the eastward movement of the low trough, there will be a rainy weather process on Fuxin $7^{\text {th }}$, which is very suitable for artificial precipitation.

At 20 o'clock on the $6^{\text {th }}$, analyze the development of the weather system and various meteorological elements, and judge that the weather system is moving slowly. The impact will be sustainable until 20:00 on the $7^{\text {th }}$. It is expected that the next 1 - 3 hours will affect the Fuxin area, which is very suitable. Carry out artificial precipitation operations. By monitoring the development and changes of radar echoes in three-dimensional systems, it can be seen that the precipitation system will mainly move from southwest to northeast, and the intensity will gradually increase. Scientific artificial precipitation will help increase precipitation and alleviate drought. Immediately declare the aircraft rain enhancement operation plan, and release the instructions for preparing the rain enhancement operation to each operation point through the communication system. According to the principle of "Beckillon process" ice water conversion, the commander learned from the satellite cloud map and radar echo data provided by the monitoring and analysis system that the echo height of the process is about $4 \mathrm{~km}$, and the area is large, and the utilization is 6 days. At 21:20, 7:03:15, 07:40, 9:22, 13:55, 16:42, etc., the integral cold water and the integral supercooled water vapor are large in the Fuxin area, and the ice crystals are small and small. It is suitable for artificial catalysis to increase the chance of ice crystal condensation tuberculosis. It adopts multi-point fan-shaped layout operation mode and multi-dose artificial precipitation operation, and receives better rainfall enhancement effect.

It can be seen from the vertical distribution of supersaturated water vapor on 
the ice surface that the $4 \mathrm{~km}$ range is moderately icing, and its supercooled water content is suitable for weather modification operations. Through the sounding comprehensive analysis and radar echo profile analysis, it can be seen that the Fuxin area is suitable for weather modification work, and the working height is $3500-4500 \mathrm{~m}$. After analyzing the multi-angle three-dimensional section around the work site, the precipitation echo height above the working point is determined. Based on the above analysis, it can be confirmed that the precipitation process on the $7^{\text {th }}$ is very much in the artificial precipitation operation, and the optimal operation height is about $4 \mathrm{~km}$. Then, the accurate working orientation and working time of each site are commanded in real time through the command system.

The system can display the real-time distribution of the weather modification operation points in Fuxin City. By clicking on any operation point, the system automatically simulates the motion trajectory of the rocket, and then superimposes the real-time 3D radar echo, which can clearly see the rocket running in the cloud, which can clearly see the rocket running in the cloud, as well as its direction and influence.

The rain-increasing operation started from 4:00 and ended at 18:00. Fuxin City dispatched a total of one rain-enhanced aircraft, 10 fire-expanding flares, 12 rocket cars, and 64 rockets, got good rain enhancement.

\section{Conclusion}

The system realizes virtual three-dimensional display of information such as weather modification work site, aircraft operation, radar echo, cloud image, automatic station, lightning, and live work through 3D GIS technology, which increases the intuitiveness and fidelity of weather modification operation command and enables the operational commander to visually analyze the changing environment of the radar echo and the impact of the terrain on the weather system. For the strong convective weather system, its evolution is closely related to the local terrain system. With the help of the strong convective weather warning of the three-dimensional geographic information system, the commander is more conducive to the judgment of the weather system change trend, and provides the scientific nature of the weather modification operation command. The development and application of a new generation of $3 \mathrm{D}$ weather modification operation command system is the first application of 3D-GIS technology in the weather modification system. It not only satisfies the work requirements of the weather modification command, but also realizes the standardized scientific management of the weather modification operation, which provides a favorable guarantee for the construction of the weather modification. At the same time, it also opened up a new page for the application of 3D-GIS technology in the meteorological industry.

\section{Conflicts of Interest}

The authors declare no conflicts of interest regarding the publication of this paper. 


\section{References}

Elsahabi, M., \& Negm, A. (2017). Building 3D Profile for Lake Nubia Using RS/GIS for Accurate Estimation of Sediment. Procedia Engineering, 181, 845-852. https://doi.org/10.1016/j.proeng.2017.02.476

Huang, X. (2004). Comprehensive Management System of Weather Modification Information in Guangxi. Guangxi Meteorology, 25, 53-55.

Huang, Y., Chen, Y., Zhou, W. et al. (2007). A Comprehensive Analysis Platform for Weather Modification Based on ArcGIS. Meteorological Monthly, 33, 116-121.

Li, Q., Zhang, Z., Li, Z., Zhang, Y., \& Zhou, S. (2017). Remote Control System of $37 \mathrm{~mm}$ Double Tube Antiaircraft Gun for Hunan Weather Modification. Meteorological \& Environmental Research, 8, 33-36.

Li, Y. (2005). Design of Weather Modification System Based on GIS Technology. Guangxi Meteorology, 26, 35-37.

Sin'kevich, A. A., Boe, B., Mikhailovskii, Y. P., Dovgalyuk, Y. A., Veremei, N. E., Gopalakrishnan, V. et al. (2018). Investigation of $\mathrm{Cu}$ Cong Seeding Effect during Rainfall Augmentation in India. Russian Meteorology and Hydrology, 43, 209-217. https://doi.org/10.3103/S1068373918040015

Strelau, J., Oniszczenko, W., Zawadzki, B., Riemann, R., \& Angleitner, A. (2013). Design and Application of a City-Level Weather Modification Operation Assisted Command and Control System in Geographic Sand Table. Meteorological \& Environmental Sciences, 6, 272-286.

Ujang, U., \& Rahman, A. (2013). Temporal Three-Dimensional Ontology for Geographical Information Science (GIS)-A Review. Journal of Geographic Information System, 5, 314-323. https://doi.org/10.4236/jgis.2013.53030

Wang, X., Wang, T., Zhang, T. et al. (2017). Application of the Regulations on Drawing of a Safe Firing Area Map in Weather Modification Operation. Meteorological \& Environmental Research, 8, 31-32.

Xiao, Y. (2007). Scheme Design of Weather Modification Operation. Sci-Tech Information Development \& Economy, 17, 271-272.

$\mathrm{Xu}, \mathrm{H} .$, \& Yin, J. (2017). Some Key Issues in Developing the Numerical Model for Artificial Weather Modification. Journal of Meteorological Research, 31, 1007-1017. https://doi.org/10.1007/s13351-017-7113-3

Zeng, X. (2006). Key Issues in Weather Modification. Meteorological Science and Technology, 1, 22-25. 